\title{
DALITZ PLOT ANALYSES OF CHARMLESS $B$ DECAYS
}

\author{
ZHITANG YU * \\ Physics Department, University of Wisconsin, Madison \\ Madison, CA 53705, United States of America
}

\begin{abstract}
We present preliminary results of maximum-likelihood Dalitz plot analyses performed by the $B A B A R$ Collaboration of the charmless hadronic decays $B^{0} \rightarrow K^{+} \pi^{-} \pi^{0}, B^{+} \rightarrow$ $K^{+} \pi^{-} \pi^{+}$, and $B^{+} \rightarrow \pi^{+} \pi^{-} \pi^{+}$. We report inclusive decay rates, as well as fractions and phases for intermediate resonant decays. We also report $C P$-violating charge asymmetries for intermediate resonant decays of neutral $B$ mesons.

Keywords: $B$ meson; Dalitz plot; amplitude analysis; charmless; CP violation; decay rate.
\end{abstract}

\section{Introduction}

Charmless three-body $B$ decays significantly broaden the understanding of $B$ meson decay mechanisms and provide additional possibilities for direct $C P$ violation searches. The $B$-meson decay to the three-body final state can proceed via intermediate resonances. These resonant states can interfere with each other and with the nonresonant three-body decay. The three-body state is unique in the search for weak phases as it is possible to determine the strong phase variation for overlapping resonances. A full Dalitz-plot analysis is necessary to correctly model this interference and to extract branching fractions $(\mathrm{BF})$.

Observations of the $B$ decays to the $K^{+} \pi^{-} \pi^{0}, K^{+} \pi^{-} \pi^{+}$, and $\pi^{+} \pi^{-} \pi^{+}$threebody final states have been reported by the CLEO, Belle, and BABAR collaborations in the quasi-two-body approximation ${ }^{1,2}$. Belle also reported a preliminary Dalitz plot analysis of the decay $B^{+} \rightarrow K^{+} \pi^{-} \pi^{+}{ }^{3}$. In this report, we present Dalitz plot amplitude analyses, using a Maximum Likelihood (ML) approach. Charge conjugation is always implied throughout this paper. Details can be found in Ref. 4.

The data were collected with the BABAR detector ${ }^{5}$ at the SLAC PEP-II asymmetric-energy $e^{+} e^{-}$storage ring. The sample consists of 213 million $B \bar{B}$ pairs, corresponding to an integrated luminosity of $193.2 \mathrm{fb}^{-1}$ collected at the $\Upsilon(4 S)$ resonance("on-resonance"), and an integrated luminosity of $16 \mathrm{fb}^{-1}$ collected about

* On Behalf of the BABAR Collaboration 
$40 \mathrm{MeV}$ below the $\Upsilon(4 S)$ ("off-resonance"). A subset of the on-resonance data that corresponds to $165.9 \mathrm{fb}^{-1}$ is used for the analysis of the charged $B$ decays.

\section{Analysis Method}

For each signal $B$ candidate, we apply particle identification for the charged tracks. The $\pi^{0}$ candidate must satisfy $0.11<m(\gamma \gamma)<0.16 \mathrm{GeV} / c^{2}$. Two kinematic variables, $\Delta E$ and $m_{\mathrm{ES}}{ }^{4}$, further suppress random combinations. Continuum $e^{+} e^{-} \rightarrow q \bar{q}$ $(q=u, d, s, c)$ events are the dominant background, and are suppressed by imposing requirements on topological event shape variables calculated in the $\Upsilon(4 S)$ rest frame ${ }^{4}$. Simulated events are used to study the background from other $B$ decays ( $B$-background). For charged $B$ decays, we veto signal $B$ candidates that have $J / \Psi$, $\psi(2 \mathrm{~S})$, or $D^{0}$. The overall selection efficiencies are around $16 \%$ for $B^{0} \rightarrow K^{+} \pi^{-} \pi^{0}$, $13 \%$ for $B^{+} \rightarrow K^{+} \pi^{-} \pi^{+}$and $\pi^{+} \pi^{-} \pi^{+}$, respectively.

The amplitude of the $B$ to three-body decay is the coherent sum of one uniform nonresonant (NR) term, and of several resonant amplitudes. Each resonant amplitude is written as a product of a Blatt-Weisskopf factor ${ }^{6}$, a spin-dependent kinematic function, and a dynamic function describing the resonance. The dynamic function is usually a relativistic Breit-Wigner form factor, with parameters fixed to world averages ${ }^{7}$ and varied for systematic studies. We use the Gounaris-Sakurai parameterization $^{8}$ for $\rho(770)$, the LASS parameterization ${ }^{9}$ for $K_{0}^{*}(1430)$, and a Flatté lineshape ${ }^{10}$ for $f_{0}(980)$. The magnitudes and the phases are free parameters. For charged $B$ meson decay, we assume no $C P$ violation.

For the $B^{0} \rightarrow K^{+} \pi^{-} \pi^{0}$ analysis, we use $m_{\mathrm{ES}}, \Delta E$, and the Dalitz plot to determine the yields and the amplitudes in one unbinned ML fit. For the charged $B$ analyses, we do two unbinned ML fits. First, we use $m_{\mathrm{ES}}$ to determine the signal fraction, $B$-background being fixed to expectation. Second, we fit the Dalitz plane to determine the amplitudes, using the events in the signal box region.

\section{Preliminary Results}

Table 1 shows the preliminary results of the nominal fits to on-resonance data. For $B^{0} \rightarrow K^{+} \pi^{-} \pi^{0}$, we also measure $\mathbf{B F}\left(B^{0} \rightarrow \bar{D}^{0} \pi^{0}\right)=(3.3 \pm 0.2 \pm 0.4) \times 10^{-4}$. All nominal fits show very good agreement with data.

The systematics uncertainties due to tracking, particle identification, neutral reconstruction, and kinematic selection, are assigned by comparing control channels in simulation and data. The uncertainties in the parameterization of the discriminating variables and of the resonant dynamics ("model systematics") are obtained by varying the parameters within their uncertainties.

\section{Summary}

Due to lack of knowledge of the final state interactions in $B$ decays, we have assumed uniform phase space for the nonresonant decay amplitude and parameterized 
Table 1. Results of the nominal fits. $\phi$ is the phase for $B^{0}$ decay and $\bar{\phi}$ for $\bar{B}^{0}$ decay.

\begin{tabular}{|c|c|c|c|}
\hline \multicolumn{4}{|c|}{$B^{0} \rightarrow K^{+} \pi^{-} \pi^{0}$, total $\mathrm{BF}=(34.9 \pm 2.1 \pm 3.9) \times 10^{-6}$} \\
\hline Mode & Fraction (\%) & $\operatorname{Phase}(\phi / \bar{\phi}$, degree $)$ & $A_{C P}$ \\
\hline$K^{*}(892)^{+} \pi^{-}$ & $10.4_{-2.0}^{+2.1} \pm 0.8$ & $138 \pm 35 / 174 \pm 42$ & $-0.25 \pm 0.17 \pm 0.02 \pm 0.02$ \\
\hline$\rho(770)^{-} K^{+}$ & $24.6_{-2.9}^{+3.6} \pm 0.6$ & 0.0 (fixed) / 0.0 (fixed) & $0.13_{-0.17}^{+0.14} \pm 0.04 \pm 0.13$ \\
\hline$K_{0}^{*}(1430)^{+} \pi^{-}$ & $32.2 \pm 3.8 \pm 9.4$ & $115 \pm 34 / 149 \pm 35$ & $-0.07 \pm 0.12 \pm 0.06 \pm 0.05$ \\
\hline$K_{0}^{*}(1430)^{0} \pi^{0}$ & $22.5 \pm 4.0 \pm 7.2$ & $-12 \pm 40 / 8 \pm 42$ & $-0.34 \pm 0.15 \pm 0.07 \pm 0.08$ \\
\hline$K^{*}(892)^{0} \pi^{0}$ & $5.8_{-1.5}^{+1.7} \pm 0.6$ & $-160 \pm 41 /-144 \pm 41$ & $-0.01_{-0.22}^{+0.24} \pm 0.07 \pm 0.11$ \\
\hline $\mathrm{NR}$ & $7.1_{-2.9}^{+3.6} \pm 0.1$ & $55 \pm 28 / 79 \pm 28$ & $-0.12_{-0.37}^{+0.36} \pm 0.20 \pm 0.07$ \\
\hline \multicolumn{4}{|c|}{$B^{+} \rightarrow K^{+} \pi^{-} \pi^{+}$, total $\mathrm{BF}=(61.4 \pm 2.4 \pm 4.5) \times 10^{-6}$} \\
\hline Mode & Fraction $(\%)$ & Phase (degree) & \\
\hline$\overline{K^{*}(892)^{0} \pi^{+}}$ & $11.4 \pm 2.0 \pm 1.5$ & 0.0 (fixed) & - \\
\hline$K_{0}^{*}(1430)^{0} \pi^{+}$ & $52.6 \pm 2.3 \pm 4.0$ & $167 \pm 6 \pm 6$ & - \\
\hline$\rho(770)^{0} K^{+}$ & $8.5 \pm 1.9 \pm 1.1$ & $49 \pm 22 \pm 20$ & - \\
\hline$f_{0}(980) K^{+}$ & $15.0 \pm 2.4 \pm 1.3$ & $-32 \pm 18 \pm 23$ & - \\
\hline$\chi_{c 0} K^{+}$ & $1.45 \pm 0.27 \pm 0.23$ & $9 \pm 19 \pm 12$ & - \\
\hline NR & $7.9 \pm 0.9 \pm 2.3$ & $29 \pm 14 \pm 14$ & - \\
\hline \multicolumn{4}{|c|}{$B^{+} \rightarrow \pi^{+} \pi^{-} \pi^{+}$, total $\mathrm{BF}=(16.2 \pm 2.1 \pm 1.3) \times 10^{-6}$} \\
\hline Mode & Fraction $(\%)$ & Phase (degree) & \\
\hline$\overline{\rho(770)^{0} \pi^{+}}$ & $58.2 \pm 2.9 \pm 6.0$ & 0.0 (fixed) & - \\
\hline$\rho(1450)^{0} \pi^{+}$ & $13.6 \pm 2.8 \pm 2.0$ & $34 \pm 22 \pm 10$ & - \\
\hline$f_{0}(980) \pi^{+}$ & $2.0 \pm 1.3 \pm 2.8$ & $140 \pm 35 \pm 11$ & - \\
\hline$f_{2}(1270) \pi^{+}$ & $14.3 \pm 2.0 \pm 1.8$ & $-154 \pm 20 \pm 10$ & - \\
\hline NR & $4.2 \pm 2.0 \pm 1.4$ & $35 \pm 32 \pm 11$ & - \\
\hline
\end{tabular}

the intermediate resonant amplitudes with knowledge obtained from non- $B$-meson experiments. The results are in agreement with those reported in Ref. 1, 2, and 3.

\section{References}

1. CLEO Collaboration, E. Eckhart et al., Phys. Rev. Lett. 89, 251801 (2002); BABAR Collaboration, B. Aubert et al., Phys. Rev. Lett. 91, 201802 (2003); Belle Collaboration, P. Chang et al., BELLE-CONF-0317, hep-ex/0406075 (2003).

2. Belle Collaboration, K. Abe et al., Phys. Rev. D 65, 092005 (2002); BABAR Collaboration, B. Aubert et al., presented at FPCP 2002, hep-ex/0206004;

BABAR Collaboration, B. Aubert et al., Phys. Rev. Lett. 91, 051810 (2003); Belle Collaboration, K. Abe et al., Phys. Rev. D 69, 012001 (2004).

3. Belle Collaboration, K. Abe et al., presented at HEP 2003, BELLE-CONF-0338.

4. BABAR Collaboration, B. Aubert et al., presented at ICHEP 2004, hep-ex/0408073 for neutral $B$ decay and hep-ex/0408032 for charged $B$ decay.

5. BABAR Collaboration, B. Aubert et al., Nucl. Instrum. Methods A479, 1-116 (2002).

6. J. Blatt and V. Weisskopf, "Theoretical Nuclear Physics", John Wiley \& Sons, New York, 1956.

7. Particle Data Group, S. Eidelman et al., Phys. Lett. B 592, 1 (2004).

8. G.J. Gounaris and J.J. Sakurai, Phys. Rev. Lett. 21, 244 (1968).

9. D. Aston et al., Nucl. Phys. B296, 493 (1988).

10. S.M. Flatté, Phys. Lett. B 63, 224 (1976). 